\title{
Submanifolds with nonpositive extrinsic curvature
}

\author{
Samuel Canevari ${ }^{1}$. Guilherme Machado de Freitas ${ }^{2}$. \\ Fernando Manfio ${ }^{3}$
}

Received: 19 February 2015 / Accepted: 12 May 2016 / Published online: 21 May 2016

(C) Fondazione Annali di Matematica Pura ed Applicata and Springer-Verlag Berlin Heidelberg 2016

\begin{abstract}
We prove that complete submanifolds, on which the weak Omori-Yau maximum principle for the Hessian holds, with low codimension and bounded by cylinders of small radius must have points rich in large positive extrinsic curvature. The lower the codimension is, the richer such points are. The smaller the radius is, the larger such curvatures are. This work unifies and generalizes several previous results on submanifolds with nonpositive extrinsic curvature.
\end{abstract}

Keywords Nonpositive extrinsic curvature · Cylindrically bounded submanifolds · Otsuki's Lemma $\cdot$ Omori-Yau maximum principle

Mathematics Subject Classification Primary 53C40 - 53C42 - Secondary 53A07 · 53A35

\section{Introduction}

The results of this article show that isometric immersions $f: M^{m} \rightarrow \tilde{M}^{n}$ with low codimension and nonpositive extrinsic curvature at any point must satisfy strong geometric conditions. The simplest result along this line is that a two-dimensional surface with nonpositive curvature in $\mathbb{R}^{3}$ cannot be compact. This is a consequence of the well-known fact that at a point of maximum of a distance function on a compact surface in $\mathbb{R}^{3}$, the Gaussian curvature must be positive. It turns out that the simple idea in the proof of this elementary fact has far-

Canevari's research was partially supported by CAPES/Brazil; Freitas's research was partially supported by CNPq/Brazil; Manfio's research was partially supported by FAPESP/Brazil, Grant 2014/01989-9.

Fernando Manfio

manfio@icmc.usp.br

1 Universidade Federal de Sergipe, Av. Ver. Olímpio Grande, Centro, Itabaiana 49500-000, Brazil

2 Instituto de Matemática Pura e Aplicada, Estrada Dona Castorina, 110, Jardim Botânico, Rio de Janeiro 22460-320, Brazil

3 Universidade de São Paulo, Av. Trabalhador São-carlense, 400, Centro, São Carlos 13560-970, Brazil 
reaching generalizations for non necessarily compact submanifolds in fairly general ambient Riemannian manifolds.

One of the main tools to extend this idea to higher dimensions and codimensions is an algebraic lemma due to Otsuki [13], which will be stated in next section. On the other hand, a key ingredient to handle the noncompact case is a maximum principle due to Omori [11] and generalized by Pigola-Rigoli-Setti [14]. Using this principle, Alías-Bessa-Dajczer [2] obtained an estimate for the mean curvature of an isometric immersion $f: M^{m} \rightarrow N^{n+l}=$ $P^{n} \times \mathbb{R}^{l}$, under some assumptions on the manifold $P^{n}$, whose projection onto the first factor is bounded, the so-called cylindrically bounded submanifolds. More recently, Alías-BessaMontenegro [3] have provided an estimate for the extrinsic curvatures of such submanifolds.

In the statement below and the sequel, $\rho$ stands for the distance function to a given reference point in $M^{m}, \log ^{(j)}$ is the $j$-th iterate of the logarithm and $t \gg 1$ means that $t$ is sufficiently large. Also $B_{P}[R]$ denotes the closed geodesic ball with radius $R$ centered at a point $o$ of $P^{n}$ and $\operatorname{inj}_{P}(o)$ is the injectivity radius of $P^{n}$ at $o$. Finally, $K_{M}(\sigma)$ denotes the sectional curvature of $M^{m}$ at a point $x \in M^{m}$ along the plane $\sigma \subset T_{x} M$, and similarly for $N^{n+l}$, $K_{f}(\sigma):=K_{M}(\sigma)-K_{N}\left(f_{*} \sigma\right)$ is the extrinsic sectional curvature of $f$ at $x$ along $\sigma$ and $K_{P}^{\mathrm{rad}}$ is the radial sectional curvatures of $P^{n}$ with respect to $o$, that is, the sectional curvatures of tangent planes to $P^{n}$ containing the vector $\operatorname{grad}^{P} r$, where $r$ is the distance function to $O$ in $P^{n}$.

Theorem 1 (Alías-Bessa-Montenegro, [3]) Let $f: M^{m} \rightarrow N^{n+l}=P^{n} \times \mathbb{R}^{l}$ be an isometric immersion with codimension $p=n+l-m<m-l$ of a complete Riemannian manifold whose scalar curvature satisfies

$$
s_{M}(x) \geq-A^{2} \rho^{2}(x) \prod_{j=1}^{J}\left(\log ^{(j)}(\rho(x))\right)^{2}, \rho(x) \gg 1,
$$

for some constant $A>0$ and some integer $J \geq 1$. Assume that $f(M) \subset B_{P}[R] \times \mathbb{R}^{l}$, with $0<R<\min \left\{\operatorname{inj}_{P}(o), \frac{\pi}{2 \sqrt{b}}\right\}$, where $\frac{\pi}{2 \sqrt{b}}$ is replaced by $+\infty$ if $b \leq 0$. If $K_{P}^{\mathrm{rad}} \leq b$ in $B_{P}[R]$, then

$$
\sup _{M} K_{f} \geq C_{b}^{2}(R)
$$

where

$$
C_{b}(t)=\left\{\begin{array}{lll}
\sqrt{b} \cot (\sqrt{b} t) & \text { if } b>0 \text { and } 0<t<\frac{\pi}{2 \sqrt{b}} \\
\frac{1}{t} & \text { if } b=0 \text { and } t>0 \\
\sqrt{-b} \operatorname{coth}(\sqrt{-b} t) & \text { if } b<0 \text { and } t>0 .
\end{array}\right.
$$

Moreover,

$$
\sup _{M} K_{M} \geq C_{b}^{2}(R)+\inf _{B_{P}[R]} K_{P} .
$$

Remark 1 The geometry of the Euclidean factor $\mathbb{R}^{l}$ plays essentially no role in the proof of the above result. Indeed, estimate (1.3) remains true if the former is replaced by any Riemannian manifold $Q^{l}$, which need not be even complete, whereas for (1.2) the only requirement is that $K_{Q}$ be bounded from above (see comment below Theorem 2).

It is worth pointing out that the codimension restriction $p<m-l$ cannot be relaxed. Actually, it implies that $n>2$ and $m>l+1$. In particular, in a three-dimensional ambient 
space $N^{3}$, that is, $n+l=3$, we have that $l=0$, and therefore $f(M) \subset B_{P}[R]$. In fact, the flat cylinder $\mathbb{S}^{1}(R) \times \mathbb{R} \subset B_{\mathbb{R}^{2}}[R] \times \mathbb{R}$ shows that the restriction $p<m-l$ is necessary.

On the other hand, estimates (1.2) and (1.3) are sharp. Indeed, the function $C_{b}$ is well known: the geodesic sphere $\partial B_{\mathbb{Q}_{b}^{m}}(R)$ of radius $R$ in the simply connected complete space form $\mathbb{Q}_{b}^{m}$ of constant sectional curvature $b$, with $R<\frac{\pi}{2 \sqrt{b}}$ if $b>0$, is an umbilical hypersurface with principal curvatures being precisely $C_{b}(R)$. It shows that its extrinsic and intrinsic sectional curvatures are constant and equal to $C_{b}^{2}(R)$ and $C_{b}^{2}(R)+b$, respectively, the latter following from the former by the Gauss equation. Then, for every $n>2$ and $l \geq 0$ we can consider $M^{m-1+l}=\partial B_{\mathbb{Q}_{b}^{m}}(R) \times \mathbb{R}^{l}$ and take $f: M^{m-1+l} \rightarrow B_{\mathbb{Q}_{b}^{m}}[R] \times \mathbb{R}^{l}$ to be the canonical isometric embedding. Therefore $\sup _{M} K_{f}$ and $\sup _{M} K_{M}$ are the constant extrinsic and intrinsic sectional curvatures $C_{b}^{2}(R)$ and $C_{b}^{2}(R)+b$ of $\partial B_{\mathbb{Q}_{b}^{m}}(R)$, respectively.

The purpose of this paper is to provide a more accurate conclusion than the one of Theorem 1 by precising how much extrinsic (respectively, intrinsic) sectional curvature satisfying estimate (1.2) [(respectively (1.3)] appears depending on how low the codimension is. The idea is that the lower the codimension is, the more extrinsic (respectively, intrinsic) sectional curvature satisfying (1.2) [(respectively (1.3)] will appear. Our main result can be stated as follows.

Theorem 2 Let $f: M^{m} \rightarrow N^{n+l}=P^{n} \times Q^{l}$ be an isometric immersion with codimension $p=n+l-m<m-l$ of a complete Riemannian manifold whose radial sectional curvatures satisfy

$$
K_{M}^{\mathrm{rad}}(x) \geq-A^{2} \rho^{2}(x) \prod_{j=1}^{J}\left(\log ^{(j)}(\rho(x))\right)^{2}, \rho(x) \gg 1,
$$

for some constant $A>0$ and some integer $J \geq 1$. Assume that $f(M) \subset B_{P}[R] \times Q^{l}$, with $0<R<\min \left\{\operatorname{inj}_{P}(o), \frac{\pi}{2 \sqrt{b}}\right\}$, where $\frac{\pi}{2 \sqrt{b}}$ is replaced by $+\infty$ if $b \leq 0$. If $K_{P}^{\mathrm{rad}} \leq b$ in $B_{P}[R]$, then

$$
\sup _{M} \min \left\{\max _{\sigma \subset W} K_{f}(\sigma): \operatorname{dim} W>p+l\right\} \geq C_{b}^{2}(R) .
$$

Moreover,

$$
\sup _{M} \min \left\{\max _{\sigma \subset W} K_{M}(\sigma): \operatorname{dim} W>p+l\right\} \geq C_{b}^{2}(R)+\inf _{B_{P}[R]} K_{P} .
$$

The estimates of Theorem 2 are clearly better than the ones of Theorem 1. Actually, (1.5) and (1.6) reduce to (1.2) and (1.3), respectively, only in the case of the highest allowed codimension $p=m-1-l$. On the other hand, although we make a stronger assumption on the curvature of $M^{m}$, if (1.1) holds but (1.4) does not, then, since the scalar curvature is an average of sectional curvatures, we have that $\sup _{M} K_{M}=+\infty$, and hence (1.3) is trivially satisfied. Moreover, $K_{P}$ is clearly bounded in $B_{P}[R]$, thus if also $K_{Q}$ is bounded from above, we conclude that $\sup _{M} K_{f}=+\infty$ by the Gauss equation, so that (1.2) also holds trivially in this case. Finally, note that the same example considered below Theorem 1 shows that our estimates (1.5) and (1.6) are also sharp.

Remark 2 Theorem 2 is a special case of the much stronger result, Theorem 12, given in Sect. 3. 
Let $f: M^{m} \rightarrow N^{n+l}=P^{n} \times Q^{l}$ be an isometric immersion. Recall that $f$ is said to be cylindrically bounded if there exists a (closed) geodesic ball $B_{P}[R]$ of $P^{n}$, centered at a point $o \in P^{n}$ with radius $R>0$, such that

$$
f(M) \subset B_{P}[R] \times Q^{l} .
$$

Otherwise, we say that $f$ is cylindrically unbounded. Denote by $R_{f}$ the extrinsic radius of a cylindrically bounded isometric immersion $f$ (from $o$ ), that is, the smallest $R$ for which (1.7) holds. As a consequence of Theorem 2, we have the following versions of the extrinsic radius results of Alías-Bessa-Montenegro [3].

Corollary 1 Let $f: M^{m} \rightarrow N^{n+l}=P^{n} \times Q^{l}$ be an isometric immersion with codimension $p=n+l-m<m-l$ of a complete Riemannian manifold whose radial sectional curvatures satisfy (1.4). Assume that $P^{n}$ is a complete Riemannian manifold with a pole and radial sectional curvatures $K_{P}^{\mathrm{rad}} \leq b \leq 0$. If $f$ is cylindrically bounded, then

$$
\sup _{M} \min \left\{\max _{\sigma \subset W} K_{f}(\sigma): \operatorname{dim} W>p+l\right\}>-b
$$

and the extrinsic radius satisfies

$$
R_{f} \geq C_{b}^{-1}\left(\sqrt{\sup _{M} \min \left\{\max _{\sigma \subset W} K_{f}(\sigma): \operatorname{dim} W>p+l\right\}}\right) .
$$

In particular, if

$$
\sup _{M} \min \left\{\max _{\sigma \subset W} K_{f}(\sigma): \operatorname{dim} W>p+l\right\} \leq-b,
$$

then $f$ is cylindrically unbounded.

Corollary 2 Let $f: M^{m} \rightarrow N^{n+l}=\mathbb{S}^{n} \times Q^{l}$ be an isometric immersion with codimension $p=n+l-m<m-l$ of a complete Riemannian manifold whose radial sectional curvatures satisfy (1.4). If

$$
\sup _{M} \min \left\{\max _{\sigma \subset W} K_{M}(\sigma): \operatorname{dim} W>p+l\right\} \leq 1,
$$

then

$$
R_{f} \geq \frac{\pi}{2}
$$

On the other hand, a sharp lower bound for the Ricci curvature of bounded complete Euclidean hypersurfaces was obtained by Leung [8] and extended by Veeravalli [21] to nonflat ambient space forms.

Theorem 3 (Veeravalli, [21]) Let $f: M^{n} \rightarrow \mathbb{Q}_{b}^{n+1}$ be a complete hypersurface with sectional curvature bounded away from $-\infty$ such that $f(M) \subset B_{\mathbb{Q}_{b}^{n+1}}[R]$, with $R<\frac{\pi}{2 \sqrt{b}}$ if $b>0$. Then

$$
\sup _{M} \operatorname{Ric}_{M} \geq C_{b}^{2}(R)+b,
$$

where $\operatorname{Ric}_{M}$ is the Ricci curvature of $M^{n}$. 
Theorem 2 also gives an improvement of the above result, where we consider hypersurfaces of much more general ambient spaces and obtain that estimate (1.10) actually holds for the scalar curvature. This shows the unifying character of our result.

Corollary 3 Let $f: M^{n} \rightarrow P^{n+1}$ be a complete hypersurface whose radial sectional curvatures satisfy (1.4). Assume that $f(M) \subset B_{P}[R]$, with $R$ as in Theorem 2. If $K_{P}^{\mathrm{rad}} \leq b$ in $B_{P}[R]$, then

$$
\sup _{M} s_{M} \geq C_{b}^{2}(R)+\inf _{B_{P}[R]} K_{P}
$$

Again observe that for the geodesic sphere $M^{n}=\partial_{\mathbb{Q}_{b}^{n+1}}(R)$ of radius $R$ in $\mathbb{Q}_{b}^{n+1}$ the above inequality is in fact an equality. Corollary 3 leads to similar extrinsic radius results to Corollaries 1 and 2 and, in particular, a criterion of unboundness:

Corollary 4 Let $f: M^{n} \rightarrow P^{n+1}$ be a complete hypersurface whose radial sectional curvatures satisfy (1.4). Assume that $P^{n+1}$ is a complete Riemannian manifold with a pole and sectional curvatures $K_{P} \geq c$ and $K_{P}^{\mathrm{rad}} \leq b \leq 0$. If $f(M)$ is bounded, then $\sup _{M} s_{M}>c-b$ and

$$
R_{f} \geq C_{b}^{-1}\left(\sqrt{\sup _{M} s_{M}-c}\right) .
$$

In particular, if $\sup _{M} s_{M} \leq c-b$, then $f(M)$ is unbounded.

Corollary 5 Let $f: M^{n} \rightarrow \mathbb{S}^{n+1}$ be a complete hypersurface whose radial sectional curvatures satisfy (1.4). If $\sup _{M} s_{M} \leq 1$, then

$$
R_{f} \geq \frac{\pi}{2} .
$$

Finally, we also generalize in the same spirit of Theorem 2 the second part of the work of Alías-Bessa-Montenegro [3], concerning proper complete cylindrically bounded submanifolds with the norm of the second fundamental form with certain controlled growth.

Theorem 4 Let $f: M^{m} \rightarrow N^{n+l}=P^{n} \times Q^{l}$ be a proper isometric immersion with codimension $p=n+l-m<m-l$ of a complete Riemannian manifold. Assume that $f(M) \subset B_{P}[R] \times Q^{l}$, with $R$ as in Theorem 2. If $K_{P}^{\mathrm{rad}} \leq b$ in $B_{P}[R], Q^{l}$ is a complete Riemannian manifold with a pole and

$$
\sup _{f^{-1}\left(B_{P}[R] \times \partial B_{Q}(t)\right)}\|\alpha\| \leq \varsigma(t),
$$

where $\alpha$ is the second fundamental form of $f$ and $\varsigma:[0,+\infty) \rightarrow \mathbb{R}$ is a positive function satisfying $\int_{0}^{+\infty} 1 / \varsigma=+\infty$, then (1.5) and (1.6) hold.

For hypersurfaces, the growth rate of the norm of the second fundamental form can be improved as follows.

Theorem 5 Let $f: M^{n} \rightarrow N^{n+1}=P^{n+1-l} \times Q^{l}, n-l>1$, be a properly immersed complete hypersurface with $f(M) \subset B_{P}[R] \times Q^{l}$, with $R$ as in Theorem 2. Suppose that $N^{n+1}$ satisfies the assumptions as in Theorem 4 and the second fundamental form $\alpha$ satisfies

$$
\sup _{f^{-1}\left(B_{P}[R] \times \partial B_{Q}(t)\right)}\|\alpha\| \leq \varsigma^{2}(t),
$$


where $\varsigma:[0,+\infty) \rightarrow \mathbb{R}$ is a positive function satisfying

$$
\int_{0}^{+\infty} \frac{1}{\varsigma}=+\infty \text { and } \limsup _{t \rightarrow+\infty} \frac{1}{\varsigma(t)}<+\infty
$$

Then (1.5) and (1.6) hold.

\section{Preliminaries}

Our main tools to build the proof of Theorem 2 are Otsuki's Lemma, the Omori-Yau maximum principle and the Hessian comparison theorem, which for the sake of organization will be presented in two subsections.

\subsection{Otsuki's Lemma}

Throughout this subsection, $V^{n}$ and $W^{p}$ will be real vector spaces of dimensions $n$ and $p$, respectively, endowed with positive definite inner products. For a symmetric bilinear form $\alpha: V^{n} \times V^{n} \rightarrow W^{p}$, we denote

$$
K_{\alpha}(X, Y)=\langle\alpha(X, X), \alpha(Y, Y)\rangle-\|\alpha(X, Y)\|^{2},
$$

for any pair of vectors $X, Y \in V^{n}$. If $\sigma$ is a two-dimensional subspace of $V^{n}$, we define

$$
K_{\alpha}(\sigma)=\frac{K_{\alpha}(X, Y)}{\|X \wedge Y\|^{2}},
$$

where $\{X, Y\}$ is any basis of $\sigma$ and $\|X \wedge Y\|^{2}=\|X\|^{2}\|Y\|^{2}-\langle X, Y\rangle^{2}$. Given an isometric immersion $f: M^{m} \rightarrow \tilde{M}^{n}$ with second fundamental form $\alpha$, then for any $x \in M^{m}$ and any plane $\sigma \subset T_{x} M$ the Gauss equation yields

$$
K_{\alpha}(\sigma)=K_{f}(\sigma) .
$$

A basic tool in this article is the following algebraic lemma, known as Otsuki's Lemma (for a proof see, for instance, [7]).

Lemma 1 Let $\alpha: V^{n} \times V^{n} \rightarrow W^{p}$ be a symmetric bilinear form. Suppose there exists a real number $\lambda \geq 0$ such that

(i) $K_{\alpha}(\sigma) \leq \lambda$ for every plane $\sigma \subset V^{n}$,

(ii) $\|\alpha(X, X)\|>\sqrt{\lambda}$ for every unit vector $X \in V^{n}$.

Then $p \geq n$.

Given a symmetric bilinear form $\alpha: V^{n} \times V^{n} \rightarrow W^{p}$, a vector $X \in V^{n}$ is said to be an asymptotic vector of $\alpha$ if

$$
\alpha(X, X)=0 .
$$

In the next statement and the sequel, we write $K_{\alpha} \leq 0$ (respectively, $K_{\alpha}<0$ ) as a shorthand for $K_{\alpha}(\sigma) \leq 0$ (respectively, $K_{\alpha}(\sigma)<0$ ) for any plane $\sigma \subset V^{n}$.

Corollary 6 Let $\alpha: V^{n} \times V^{n} \rightarrow W^{p}$ be a symmetric bilinear form.

(i) If $K_{\alpha} \leq 0$, then any subspace $S \subset V^{n}$, with $\operatorname{dim} S>p$, contains a nonzero asymptotic vector of $\alpha$. 
(ii) If $K_{\alpha}<0$, then $p \geq n-1$.

Proof (i) This is just an equivalent way of stating Lemma 1 for $\lambda=0$.

(ii) If there are no nonzero asymptotic vectors of $\alpha$, the result follows from Lemma 1. Suppose $p<n-1$, and assume that there exists a nonzero vector $X_{0} \in V^{n}$ such that $\alpha\left(X_{0}, X_{0}\right)=0$. Denote by $U$ the orthogonal complement to $X_{0}$ in $V^{n}$, and consider the linear map $B_{X_{0}}: U \rightarrow W^{p}$ defined by $B_{X_{0}}(Y)=\alpha\left(X_{0}, Y\right)$. Since $\operatorname{dim} U=n-1>p$, there exists a nonzero vector $Y_{0} \in U$ such that $B_{X_{0}}\left(Y_{0}\right)=0$. This fact, together with $\alpha\left(X_{0}, X_{0}\right)=0$, contradicts the assumption.

The following result is a direct consequence of Corollary 6-(ii).

Theorem 6 Let $f: M^{n} \rightarrow \tilde{M}^{n+p}$ be an isometric immersion. Assume that there exists a point $x_{0} \in M^{n}$ and a subspace $V_{x_{0}} \subset T_{x_{0}} M$ with dimensiond such that $K_{f}(\sigma)<0$ along every plane $\sigma \subset V_{x_{0}}$. Then $p \geq d-1$.

The preceding inequality is sharp, as shown, e.g., by the $n$-dimensional Clifford torus in $\mathbb{S}^{2 n-1}$. Theorem 6 comes from a purely algebraic restriction on the "codimension" $p$ of symmetric bilinear forms $\alpha: V^{n} \times V^{n} \rightarrow W^{p}$ with $K_{\alpha}<0$, which gives its punctual nature. If, on the other hand, $K_{f}(\sigma) \leq 0$ in the above statement, it is possible to use part ( $i$ ) of Corollary 6 to obtain the stronger restriction $p \geq d$, provided that the subspace $V_{x_{0}}$ is free of asymptotic directions. Actually, this is a central idea in the study of the global implications of nonpositive extrinsic curvature in low codimension. The presence of a certain amount of nonpositive extrinsic sectional curvature everywhere together with some global assumption that can guarantee the existence of points without asymptotic directions must imply codimension restrictions. For compact Riemannian manifolds, for instance, one obtains the following generalization of a result for the flat case due to Tompkins [20].

Theorem 7 Let $M^{n}$ be a compact Riemannian manifold such that at any point $x \in M^{n}$ there exists a subspace $V_{x}$ of $T_{x} M$ with dimension d such that $K_{M}(\sigma) \leq 0$ for every plane $\sigma \subset V_{x}$. If $f: M^{n} \rightarrow \mathbb{R}^{n+p}$ is an isometric immersion, then $p \geq d$.

Proof Since $M^{n}$ is compact, it is well known that there exist a point $x_{0} \in M^{n}$ and a normal vector $\xi \in N_{f} M\left(x_{0}\right)$ such that the shape operator $A_{\xi}$ is positive definite, and in particular $\alpha_{x_{0}}(X, X) \neq 0$ for every nonzero vector $X \in T_{x_{0}} M$. Furthermore, $K_{\alpha_{x_{0}}}(\sigma) \leq 0$ for every plane $\sigma \subset V_{x_{0}}$ by the Gauss equation. The statement then follows from Corollary 6- $(i)$.

For the noncompact case, on the other hand, we have the following immediate consequence of our Corollary 1.

Corollary 7 Let $f: M^{n} \rightarrow P^{n+p}$ be an isometric immersion of a complete Riemannian manifold whose radial sectional curvatures satisfy (1.4) into a Hadamard manifold. Assume that at any point $x \in M^{n}$ there exists a subspace $V_{x}$ of $T_{x} M$ with dimension $d$ such that $K_{f}(\sigma) \leq 0$ for every plane $\sigma \subset V_{x}$. If $p<d$, then $f(M)$ is unbounded.

\subsection{Omori-Yau maximum principle and Hessian comparison theorem}

The Omori-Yau maximum principle for the Hessian is said to hold on a given Riemannian manifold $M^{n}$ if, for any function $g \in C^{2}(M)$ with $g^{*}=\sup _{M} g<+\infty$, there exists a sequence of points $\left\{x_{k}\right\}_{k \in \mathbb{N}}$ in $M^{n}$ satisfying: 
(i) $g\left(x_{k}\right)>g^{*}-\frac{1}{k}$,

(ii) $\left\|\operatorname{grad}^{M} g\left(x_{k}\right)\right\|<\frac{1}{k}$,

(iii) $\operatorname{Hess}^{M} g\left(x_{k}\right)(X, X) \leq \frac{1}{k}\langle X, X\rangle$ for all $X \in T_{x_{k}} M$.

Such a sequence $\left\{x_{k}\right\}_{k \in \mathbb{N}}$ satisfying (i)-(iii) above is called an Omori-Yau Hessian sequence for $g$. One says that the Omori-Yau maximum principle holds if the condition on the Hessian is replaced by a similar one on the Laplacian, namely, if

(iii) $\Delta^{M} g\left(x_{k}\right) \leq \frac{1}{k}$.

In this case, $\left\{x_{k}\right\}_{k \in \mathbb{N}}$ is called an Omori-Yau sequence for $g$.

The following is a function theoretic characterization of Riemannian manifolds that satisfy the Omori-Yau maximum principle for the Hessian. For the proof of this, as well as of the other results related to the Omori-Yau maximum principle in this subsection, we refer to [14].

Theorem 8 Assume that the Riemannian manifold $M^{n}$ supports a nonnegative function $\gamma \in C^{2}(M)$ satisfying the following conditions:

(a) $\gamma$ is proper, that is, $\gamma(x) \rightarrow+\infty$ as $x \rightarrow \infty$,

(b) $\left\|\operatorname{grad}^{M} \gamma\right\| \leq c \sqrt{\gamma}$ outside a compact subset of $M^{n}$ for some constant $c>0$,

(c) Hess $^{M} \gamma \leq d \sqrt{\gamma F(\sqrt{\gamma})}\langle$, $\rangle$ outside a compact subset of $M^{n}$, for some $d>0$ and some $F \in C^{\infty}([0,+\infty))$ that satisfies:

$$
\text { (i) } F(0)>0 \text {, (ii) } F^{\prime}(t) \geq 0 \text { on }[0,+\infty) \text {, (iii) } 1 / \sqrt{F(t)} \notin L^{1}[0,+\infty) \text {. }
$$

Then, the Omori-Yau maximum principle for the Hessian holds on $M^{n}$.

We point out that, although completeness of $M^{n}$ is not required in Theorem 8 , it is a consequence of the assumptions $(a)$ and $(b)$. Examples of functions satisfying the requirements in Theorem 8 are given by

$$
F(t)=A^{2} t^{2} \prod_{j=1}^{J}\left(\log ^{(j)} t\right)^{2}, t \gg 1,
$$

where $A>0$ is a constant and $J \geq 1$ is an integer.

Sometimes, for the applications of the maximum principle as in our paper, the following weaker version is enough.

The weak Omori-Yau maximum principle for the Hessian is said to hold on a Riemannian manifold $M^{n}$ if for any function $g \in C^{2}(M)$ with $g^{*}=\sup _{M} g<+\infty$ there exists a sequence of points $\left\{x_{k}\right\}_{k \in \mathbb{N}}$ satisfying:

(i) $g\left(x_{k}\right)>g^{*}-\frac{1}{k}$,

(ii) $\operatorname{Hess}^{M} g\left(x_{k}\right)(X, X) \leq \frac{1}{k}\langle X, X\rangle$ for all $X \in T_{x_{k}} M$.

A sequence $\left\{x_{k}\right\}_{k \in \mathbb{N}}$ satisfying (i) and (ii) is called a weak Omori-Yau Hessian sequence for $g$.

Riemannian manifolds that satisfy the weak Omori-Yau maximum principle for the Hessian are characterized as follows.

Theorem 9 Assume that the Riemannian manifold $M^{n}$ supports a nonnegative function $\gamma \in C^{2}(M)$ satisfying the following conditions:

(a) $\gamma$ is proper, 
(b) Hess $^{M} \gamma \leq d \sqrt{\gamma F(\sqrt{\gamma})}\langle$, $\rangle$ outside a compact subset of $M^{n}$, for some $d>0$ and some $F \in C^{\infty}([0,+\infty))$ as in Theorem 8 .

Then, the weak Omori-Yau maximum principle for the Hessian holds on $M^{n}$.

Accordingly, it is said that the weak Omori-Yau maximum principle holds if (ii) is replaced by the condition

(ii) $\Delta^{M} g\left(x_{k}\right) \leq \frac{1}{k}$,

in which case $\left\{x_{k}\right\}_{k \in \mathbb{N}}$ is called a weak Omori-Yau sequence for $g$.

The function theoretic approach to the Omori-Yau Maximum Principle given in Theorem 8 allows one to apply it in different situations, where the choices of $\gamma$ and $F$ are suggested by the geometric setting. For instance, one has the following special case, where as previously agreed $\rho$ stands for the distance function on a Riemannian manifold $M^{n}$ to a fixed point.

Theorem 10 Let $M^{n}$ be a complete noncompact Riemannian manifold. Assume that $K_{M}^{\mathrm{rad}} \geq$ $-F(\rho)$, where $F \in C^{\infty}[0,+\infty)$ satisfies the conditions listed in Theorem 8 and is even at the origin, that is, its derivatives satisfy $F^{(2 k+1)}(0)=0$ for $k \geq 0$. Then, the Omori-Yau maximum principle for the Hessian holds on $M^{n}$.

Remark 3 If we only assume that $\operatorname{Ric}_{M}\left(\operatorname{grad}^{M} \rho\right) \geq-F(\rho)$, then the conclusion is that the Omori-Yau maximum principle holds on $M^{n}$.

The last ingredient for the proof of Theorem 2 is the following version of the well-known Hessian comparison theorem given in [16].

Theorem 11 Let $M^{n}$ be a Riemannian manifold and $o, x \in M^{n}$ be such that there is a minimizing geodesic $\gamma$ joining $o$ and $x$, and let $\rho$ be the distance function to o. Suppose that $K_{M}^{\mathrm{rad}} \leq b$ along $\gamma$. If $b>0$ assume $\rho(x)<\frac{\pi}{2 \sqrt{b}}$. Then, we have

$$
\operatorname{Hess}^{M} \rho \geq C_{b}(\rho)(\langle,\rangle-d \rho \otimes d \rho)
$$

along $\gamma$.

\section{Proofs}

Let $f: M^{m} \rightarrow \tilde{M}^{n}$ be an isometric immersion between Riemannian manifolds. Given a function $h \in C^{\infty}(\tilde{M})$ we set $g=h \circ f \in C^{\infty}(M)$. Since

$$
\left\langle\operatorname{grad}^{M} g(x), X\right\rangle=\left\langle\operatorname{grad}^{\tilde{M}} h(f(x)), f_{*} X\right\rangle
$$

for every $x \in M^{n}$ and $X \in T_{x} M$, we obtain

$$
f_{*} \operatorname{grad}^{M} g(x)=\left(\operatorname{grad}^{\tilde{M}} h(f(x))\right)^{T},
$$

where ()$^{T}$ is the tangent component. An easy computation using the Gauss formula gives the well-known relation (see e.g. [6]):

$$
\operatorname{Hess}^{M} g(x)(X, Y)=\operatorname{Hess}^{\tilde{M}} h(f(x))\left(f_{*} X, f_{*} Y\right)+\left\langle\operatorname{grad}^{\tilde{M}} h(f(x)), \alpha_{x}(X, Y)\right\rangle
$$


for all $x \in M^{n}$ and $X, Y \in T_{x} M$, where $\alpha_{x}$ stands for the second fundamental form of $f$ at $x$. In particular, taking traces with respect to an orthonormal frame $\left\{e_{1}, \ldots, e_{m}\right\}$ in $T_{x} M$ yields

$$
\Delta^{M} g(x)=\sum_{i=1}^{m} \operatorname{Hess}^{\tilde{M}} h(f(x))\left(f_{*} e_{i}, f_{*} e_{i}\right)+n\left\langle\operatorname{grad}^{\tilde{M}} h(f(x)), H(x)\right\rangle,
$$

where $H(x)=\frac{1}{n} \sum_{i=1}^{m} \alpha_{x}\left(e_{i}, e_{i}\right)$ is the mean curvature vector of $f$ at $x$.

Given an isometric immersion $f: M^{m} \rightarrow N^{n+l}=P^{n} \times Q^{l}$, we denote by $\pi_{P}: N^{n+l} \rightarrow$ $P^{n}$ and $\pi_{Q}: N^{n+l} \rightarrow Q^{l}$ the projections onto $P^{n}$ and $Q^{l}$, respectively. We write $(y, z)$ for points in $N^{n+l}=P^{n} \times Q^{l}$ and by abuse of notation denote $y=\pi_{P} \circ f$ and $z=\pi_{Q} \circ f$.

Moreover, set

$$
\psi_{b}=\left\{\begin{array}{lll}
1-\cos (\sqrt{b} t) & \text { if } \quad b>0, \\
t^{2} & \text { if } \quad b=0, \\
\cosh (\sqrt{-b} t) & \text { if } \quad b<0,
\end{array}\right.
$$

where $t>0$ if $b \leq 0$ and $0<t<\frac{\pi}{2 \sqrt{b}}$ if $b>0$. Hence $\psi_{b}^{\prime \prime}=C_{b} \psi_{b}^{\prime}$. We define $h \in C^{\infty}(N)$ by $h=\psi_{b} \circ r \circ \pi_{P}$, where $r$ is the distance function on $P^{n}$ to the reference point $o$. We call $g=h \circ f$ the modified radial function of $f$.

\subsection{Proofs of Theorem 2 and corollaries}

As mentioned in Remark 2, Theorem 2 is a consequence of the following stronger result. Here the algebraic codimension $p(x)$ of an isometric immersion $f: M^{m} \rightarrow \tilde{M}^{n}$ at $x \in M^{m}$ is the dimension of its first normal space $N_{1}(x)$ and a sequence of real numbers $\left\{p_{k}\right\}_{k \in \mathbb{N}}$ is said to be strictly bounded from above by another $\left\{q_{k}\right\}_{k \in \mathbb{N}}$ if $p_{k}<q_{k}$ for all $k \in \mathbb{N}$.

Theorem 12 Let $f: M^{m} \rightarrow N^{n+l}=P^{n} \times Q^{l}$ be an isometric immersion. Assume that $f(M) \subset B_{P}[R] \times Q^{l}$, with $0<R<\min \left\{\operatorname{inj}_{P}(o), \frac{\pi}{2 \sqrt{b}}\right\}$, where $\frac{\pi}{2 \sqrt{b}}$ is replaced by $+\infty$ if $b \leq 0$. If $K_{P}^{\mathrm{rad}} \leq b$ in $B_{P}[R]$, then

$$
\lim \inf \min \left\{\max _{\sigma \subset W} K_{f}(\sigma): \operatorname{dim} f_{*} W \cap T_{y\left(x_{k}\right)} P>p\left(x_{k}\right)\right\} \geq C_{b}^{2}(R)
$$

for all weak Omori-Yau Hessian sequence $\left\{x_{k}\right\}_{k \in \mathbb{N}}$ for the modified radial function of $f$ with algebraic codimension sequence $\left\{p\left(x_{k}\right)\right\}_{k \in \mathbb{N}}$ strictly bounded from above by $\left\{\operatorname{dim} f_{*} T_{x_{k}} M \cap T_{y\left(x_{k}\right)} P\right\}_{k \in \mathbb{N}}$. Furthermore,

$$
\lim \inf \min \left\{\max _{\sigma \subset W} K_{M}(\sigma): \operatorname{dim} f_{*} W \cap T_{y\left(x_{k}\right)} P>p\left(x_{k}\right)\right\} \geq C_{b}^{2}(R)+\inf _{B_{P}[R]} K_{P} .
$$

Proof By the assumption that $f(M) \subset B_{P}[R] \times Q^{l}$, the modified radial function $g$ satisfies

$$
g^{*} \leq \psi_{b}(R)<+\infty,
$$

where we write ()$^{*}=\sup _{M}()$. Let $\left\{x_{k}\right\}_{k \in \mathbb{N}}$ be a weak Omori-Yau Hessian sequence for $g$, that is,

(i) $g\left(x_{k}\right)>g^{*}-\frac{1}{k}$,

(ii) $\operatorname{Hess}^{M} g\left(x_{k}\right) \leq \frac{1}{k}\langle$,$\rangle .$ 
For each $k \in \mathbb{N}$, take a tangent subspace $W_{k} \subset T_{x_{k}} M$ such that $\operatorname{dim} V_{k}>p_{k}$, where $V_{k}=f_{*}^{-1}\left(f_{*} W_{k} \cap T_{y_{k}} P\right)$ and for simplicity of notation we write $p_{k}=p\left(x_{k}\right), y_{k}=$ $y\left(x_{k}\right)$. If the algebraic codimension sequence $\left\{p_{k}\right\}_{k \in \mathbb{N}}$ is strictly bounded from above by $\left\{\operatorname{dim} f_{*} T_{x_{k}} M \cap T_{y_{k}} P\right\}_{k \in \mathbb{N}}$, then at least $W_{k}=T_{x_{k}} M$ satisfies the latter condition, so that the sets on the left-hand side of inequalities (3.3) and (3.4) are nonempty. The idea of the argument is to use (ii) above and (3.2) to estimate $\left\|\alpha_{x_{k}}(X, X)\right\|$ for $X \in V_{k}$, and then apply Lemma 1 to $\left.\alpha_{x_{k}}\right|_{V_{k} \times V_{k}}$. This will imply the estimates in the statement. By (3.1), we have

$$
\operatorname{grad}^{N} h(f(x))=f_{*} \operatorname{grad}^{M} g(x)+\left(\operatorname{grad}^{N} h(f(x))\right)^{\perp},
$$

where ()$^{\perp}$ denotes the normal component. Note that

$$
\operatorname{grad}^{N} h(y, z)=\psi_{b}^{\prime}(r(y)) \operatorname{grad}^{P} r(y) .
$$

Since $h$ only depends on $P^{n}$, we obtain from (3.2) and (3.5) that

$$
\begin{aligned}
\operatorname{Hess}^{M} g(x)(X, X)= & \operatorname{Hess}^{P} \psi_{b} \circ r(y(x))\left(X_{P}, X_{P}\right) \\
& +\psi_{b}^{\prime}(r(y(x)))\left\langle\operatorname{grad}^{P} r(y(x)), \alpha_{x}(X, X)\right\rangle,
\end{aligned}
$$

where $X_{P}=y_{*} X$. Observe that

$$
\begin{aligned}
\operatorname{Hess}^{P} \psi_{b} \circ r(y)\left(X_{P}, X_{P}\right)= & \psi_{b}^{\prime \prime}(r(y))\left\langle\operatorname{grad}^{P} r(y), X_{P}\right\rangle^{2} \\
& +\psi_{b}^{\prime}(r(y)) \operatorname{Hess}^{P} r(y)\left(X_{P}, X_{P}\right) .
\end{aligned}
$$

Since $\psi_{b}^{\prime \prime}=C_{b} \psi_{b}^{\prime}$, the last two equations yield

$$
\begin{aligned}
\operatorname{Hess}^{M} g(x)(X, X)= & \psi_{b}^{\prime}(r(y(x)))\left(C_{b}(r(y(x)))\left\langle\operatorname{grad}^{P} r(y(x)), X_{P}\right\rangle^{2}\right. \\
& \left.+\left\langle\operatorname{grad}^{P} r(y(x)), \alpha_{x}(X, X)\right\rangle+\operatorname{Hess}^{P} r(y(x))\left(X_{P}, X_{P}\right)\right) .
\end{aligned}
$$

Theorem 11 gives

$$
\begin{aligned}
\operatorname{Hess}^{P} r(y)(Y, Y) & =\operatorname{Hess}^{P} r(y)\left(Y^{\perp}, Y^{\perp}\right) \\
& \geq C_{b}(r(y))\left(\|Y\|^{2}-\left\langle\operatorname{grad}^{P} r(y), Y\right\rangle^{2}\right),
\end{aligned}
$$

where $Y \in T_{y} P$ and here $Y^{\perp}$ is defined by the orthogonal decomposition

$$
Y=\left\langle\operatorname{grad}^{P} r(y), Y\right\rangle \operatorname{grad}^{P} r(y)+Y^{\perp} .
$$

Now, since $X_{P}=f_{*} X$ for any $X \in V_{k}$, we obtain from (3.6) and (3.7) that

$$
\begin{aligned}
\operatorname{Hess}^{M} g\left(x_{k}\right)(X, X) & \geq \psi_{b}^{\prime}\left(r_{k}\right)\left(C_{b}\left(r_{k}\right)\|X\|^{2}+\left\langle\operatorname{grad}^{P} r\left(y_{k}\right), \alpha_{x_{k}}(X, X)\right\rangle\right) \\
& \geq \psi_{b}^{\prime}\left(r_{k}\right)\left(C_{b}\left(r_{k}\right)\|X\|^{2}-\left\|\alpha_{x_{k}}(X, X)\right\|\right),
\end{aligned}
$$

where $r_{k}=r\left(y_{k}\right)$. Hence, by $(i i)$

$$
\frac{1}{k}\|X\|^{2} \geq \psi_{b}^{\prime}\left(r_{k}\right)\left(C_{b}\left(r_{k}\right)\|X\|^{2}-\left\|\alpha_{x_{k}}(X, X)\right\|\right)
$$

for every $x_{k}$ and every $X \in V_{k}$, and therefore,

$$
\left\|\alpha_{x_{k}}(X, X)\right\| \geq\left(C_{b}\left(r_{k}\right)-\frac{1}{k \psi_{b}^{\prime}\left(r_{k}\right)}\right)\|X\|^{2} .
$$


Since $g\left(x_{k}\right)=\psi_{b}\left(r_{k}\right)$ approaches $g^{*}=\psi_{b}\left(r^{*}\right)>0$ by $(i)$ and $\left.\psi_{b}\right|_{[0, R]}$ is a homeomorphism onto its image (recall that $R<\frac{\pi}{2 \sqrt{b}}$ if $b>0$ ), it follows that $r_{k}$ goes to $r^{*}>0$, and in particular $\psi_{b}^{\prime}\left(r_{k}\right) \rightarrow \psi_{b}^{\prime}\left(r^{*}\right)>0$. Thus,

$$
C_{b}\left(r_{k}\right)-\frac{1}{k \psi_{b}^{\prime}\left(r_{k}\right)}>0
$$

for $k$ sufficiently large and, as $\operatorname{dim} V_{k}>p_{k}$, we can apply Lemma 1 to

$$
\left.\alpha_{x_{k}}\right|_{V_{k} \times V_{k}}: V_{k} \times V_{k} \rightarrow N_{1}\left(x_{k}\right) .
$$

We obtain a plane $\sigma_{k} \subset V_{k}$ such that, by the Gauss equation,

$$
K_{f}\left(\sigma_{k}\right)=K_{\alpha_{x_{k}}}\left(\sigma_{k}\right) \geq\left(C_{b}\left(r_{k}\right)-\frac{1}{k \psi_{b}^{\prime}\left(r_{k}\right)}\right)^{2} .
$$

In particular,

$$
\max _{\sigma \subset W_{k}} K_{f}(\sigma) \geq\left(C_{b}\left(r_{k}\right)-\frac{1}{k \psi_{b}^{\prime}\left(r_{k}\right)}\right)^{2},
$$

but since the subspaces $W_{k} \subset T_{x_{k}} M$ satisfying $\operatorname{dim} f_{*}\left(W_{k}\right) \cap T_{y_{k}} P>p_{k}$ have been taken arbitrarily, we have indeed

$$
\min \left\{\max _{\sigma \subset W_{k}} K_{f}(\sigma): \operatorname{dim} f_{*} W_{k} \cap T_{y_{k}} P>p_{k}\right\} \geq\left(C_{b}\left(r_{k}\right)-\frac{1}{k \psi_{b}^{\prime}\left(r_{k}\right)}\right)^{2} .
$$

Then (3.3) follows by letting $k \rightarrow+\infty$. We will now compare the sectional curvatures $K_{M}\left(\sigma_{k}\right)$ and $K_{N}\left(f_{*} \sigma_{k}\right)$. Since $\sigma_{k} \subset V_{k} \perp T_{z_{k}} Q, z_{k}=z\left(x_{k}\right)$, then

$$
K_{N}\left(f_{*} \sigma_{k}\right)=K_{P}\left(y_{*} \sigma_{k}\right) \text {. }
$$

Then, we have that

$$
\begin{aligned}
K_{M}\left(\sigma_{k}\right) & =K_{f}\left(\sigma_{k}\right)+K_{P}\left(y_{*} \sigma_{k}\right) \\
& \geq\left(C_{b}\left(r_{k}\right)-\frac{1}{k \psi_{b}^{\prime}\left(r_{k}\right)}\right)^{2}+\inf _{B_{P}[R]} K_{P},
\end{aligned}
$$

and (3.4) follows by a similar argument.

Remark 4 That the maximum and minimum on the left-hand side of (3.3) (and similarly for (3.4)) are indeed attained can be argued as follows. At each $x=x_{k}$, the extrinsic sectional curvature $K_{f}=K_{\alpha_{x}}: G_{2}\left(T_{x} M\right) \rightarrow \mathbb{R}$ is a continuous function on the Grassmannian $G_{2}\left(T_{x} M\right)$ of (nonoriented) planes in $T_{x} M$, and by compactness attains its maximum and minimum. Since $G_{2}(W)$ is a compact subset of $G_{2}\left(T_{x} M\right)$ for any subspace $W \subset T_{x} M$, so does the restriction $\left.K_{f}\right|_{W}$. Let

$$
\left\{W_{j}\right\}_{j \in \mathbb{N}} \subset \mathcal{W}:=\left\{W \subset T_{x} M: \operatorname{dim} f_{*} W \cap T_{y(x)} P>p(x)\right\} \neq \emptyset
$$

be a sequence such that

$$
\max _{\sigma \subset W_{j}} K_{f}(\sigma) \rightarrow \inf \left\{\max _{\sigma \subset W} K_{f}(\sigma): W \in \mathcal{W}\right\},
$$

as $j \rightarrow+\infty$. After passing to a subsequence we can without loss of generality assume that all $W_{j}$ have the same dimension $d$ and converge to some $W_{\infty} \in G_{d}\left(T_{x} M\right)$, where 
$G_{d}\left(T_{x} M\right)$ denotes the Grassmannian of (nonoriented) $d$-planes in $T_{x} M$. Moreover, since $f_{*}$ is an isomorphism onto its image, it is clear that the function $W \in G_{d}\left(T_{x} M\right) \mapsto \operatorname{dim} f_{*} W \cap$ $T_{y(x)} P$ is upper semicontinuous, and so

$$
\operatorname{dim} f_{*}\left(W_{\infty}\right) \cap T_{y(x)} P>p(x),
$$

or equivalently, $W_{\infty} \in \mathcal{W}$. Hence,

$$
\max _{\sigma \subset W_{\infty}} K_{f}(\sigma) \in\left\{\max _{\sigma \subset W} K_{f}(\sigma): W \in \mathcal{W}\right\} .
$$

Finally, a straightforward contradiction argument allows to conclude that

$$
\lim _{j \rightarrow+\infty} \max _{\sigma \subset W_{j}} K_{f}(\sigma)=\max _{\sigma \subset W_{\infty}} K_{f}(\sigma),
$$

and therefore

$$
\max _{\sigma \subset W_{\infty}} K_{f}(\sigma)=\min \left\{\max _{\sigma \subset W} K_{f}(\sigma): W \in \mathcal{W}\right\} .
$$

Proof of Theorem 2 According to Theorem 10, the curvature decay in the statement is sufficient to conclude that the Omori-Yau maximum principle for the Hessian holds on $M^{m}$. Thus there exists in $M^{m}$ an Omori-Yau Hessian sequence $\left\{x_{k}\right\}_{k \in \mathbb{N}}$ for the modified radial function of $f$, whose algebraic codimension sequence $\left\{p_{k}\right\}_{k \in \mathbb{N}}$ satisfies

$$
p_{k} \leq p<m-l \leq \operatorname{dim} f_{*} T_{x_{k}} M \cap T_{y_{k}} P,
$$

that is, $\left\{p_{k}\right\}_{k \in \mathbb{N}}$ is strictly bounded from above by $\left\{\operatorname{dim} f_{*} T_{x_{k}} M \cap T_{y_{k}} P\right\}$ as required in Theorem 12. Moreover, given a subspace $W \subset T_{x_{k}} M$ with $\operatorname{dim} W>p+l$, it is clear that $\operatorname{dim} f_{*} W \cap T_{y_{k}} P>p \geq p_{k}$. In other words,

$$
\left\{W \subset T_{x_{k}} M: \operatorname{dim} W>p+l\right\} \subset\left\{W \subset T_{x_{k}} M: \operatorname{dim} f_{*} W \cap T_{y_{k}} P>p_{k}\right\},
$$

and in particular

$$
\left\{\max _{\sigma \subset W} K_{f}(\sigma): \operatorname{dim} W>p+l\right\} \subset\left\{\max _{\sigma \subset W} K_{f}(\sigma): \operatorname{dim} f_{*} W \cap T_{y_{k}} P>p_{k}\right\} .
$$

Therefore,

$$
\min \left\{\max _{\sigma \subset W} K_{f}(\sigma): \operatorname{dim} W>p+l\right\} \geq \min \left\{\max _{\sigma \subset W} K_{f}(\sigma): \operatorname{dim} f_{*} W \cap T_{y_{k}} P>p_{k}\right\},
$$

and (1.5) follows immediately from (3.3). Similarly, (1.6) follows from (3.4).

Proof of Corollary 1 Follows immediately from (1.5) observing that inf $C_{b}=\sqrt{-b}$ for $b \leq 0$.

Remark 5 If, in addition, $K_{P} \geq c$ in Corollary 1, then (1.6) provides

$$
\sup _{M} \min \left\{\max _{\sigma \subset W} K_{M}(\sigma): \operatorname{dim} W>p+l\right\} \leq c-b
$$

as a criterion of cylindrical unboundness. In particular, when $P^{n+p}$ is either the Euclidean space $\mathbb{R}^{n+p}$ or the hyperbolic space $\mathbb{H}^{n+p}$ and $M^{n}$ is a complete Riemannian manifold [(whose radial sectional curvatures satisfy (1.4)] in which at any point $x \in M^{n}$ there exists a subspace $V_{x}$ of $T_{x} M$ with dimension $d$ such that $K_{M}(\sigma) \leq 0$ for every plane $\sigma \subset V_{x}$, we conclude that every isometric immersion $f: M^{n} \rightarrow P^{n+p}$ with codimension $p<d$ is unbounded (compare with Corollary 7). 
Proof of Corollary 3 Here $p=1$ and $l=0$, so that (1.6) in Theorem 2 yields

$$
\sup _{M} \min K_{M} \geq C_{b}^{2}(R)+\inf _{B_{P}[R]} K_{P} .
$$

Since clearly $s_{M} \geq \min K_{M}$ at each point, the corollary follows.

\subsection{Proofs of Theorems 4 and 5}

Proof of Theorem 4 Again consider the modified radial function $g: M^{m} \rightarrow \mathbb{R}$. Since $\pi_{P}(f(M)) \subset B_{P}[R]$, we have that $g^{*} \leq \psi_{b}(R)$. Let $\phi: M^{m} \rightarrow[0,+\infty)$ be given by

$$
\phi(x)=\exp \left(\int_{0}^{|z(x)|} \frac{\mathrm{d} s}{\varsigma(s)}\right),
$$

where II stands for the distance function to the pole of $Q^{l}$. Since $f$ is proper and $\pi_{P}(f(M)) \subset$ $B_{P}[R]$, then the function $|z(x)|$ satisfies $|z(x)| \rightarrow+\infty$ as $x \rightarrow \infty$. By hypothesis we have that $\int_{0}^{+\infty} 1 / \varsigma(s) \mathrm{d} s=+\infty$ so that $\phi(x) \rightarrow+\infty$ as $x \rightarrow \infty$. We let $x_{0} \in M^{m}$ with $\pi_{P}\left(f\left(x_{0}\right)\right) \neq o$ and set

$$
g_{k}(x)=\frac{g(x)-g\left(x_{0}\right)+1}{\phi(x)^{1 / k}} .
$$

Thus $g_{k}\left(x_{0}\right)>0$, and since $g^{*} \leq \psi_{b}(R)<+\infty$ and $\phi(x) \rightarrow+\infty$ as $x \rightarrow \infty$, we have that $\lim \sup _{x \rightarrow \infty} g_{k}(x) \leq 0$. Hence $g_{k}$ attains a positive absolute maximum at a point $x_{k} \in M^{m}$. This procedure yields a sequence $\left\{x_{k}\right\}_{k \in \mathbb{N}}$ such that (passing to a subsequence if necessary) $g\left(x_{k}\right)$ converges to $g^{*}$. First suppose that $x_{k} \rightarrow \infty$ as $k \rightarrow+\infty$. Since $g_{k}$ attains a maximum at $x_{k}$, we have $\operatorname{grad}^{M} g_{k}\left(x_{k}\right)=0$ and $\operatorname{Hess}^{M} g_{k}\left(x_{k}\right)(X, X) \leq 0$ for every $X \in T_{x_{k}} M$. This yields

$$
\operatorname{grad}^{M} g\left(x_{k}\right)=\frac{g\left(x_{k}\right)-g\left(x_{0}\right)+1}{k \phi\left(x_{k}\right)} \operatorname{grad}^{M} \phi\left(x_{k}\right)
$$

and

$$
\begin{aligned}
\operatorname{Hess}^{M} g\left(x_{k}\right) & \leq \frac{g\left(x_{k}\right)-g\left(x_{0}\right)+1}{k \phi\left(x_{k}\right)}\left(\operatorname{Hess}^{M} \phi\left(x_{k}\right)+\left(\frac{1}{k}-1\right) \frac{1}{\phi\left(x_{k}\right)} \mathrm{d} \phi \otimes \mathrm{d} \phi\right) \\
& \leq \frac{g\left(x_{k}\right)-g\left(x_{0}\right)+1}{k \phi\left(x_{k}\right)} \operatorname{Hess}^{M} \phi\left(x_{k}\right) .
\end{aligned}
$$

Since $\phi(x)=\zeta(z(x))$, where $\zeta(z)=\exp \left(\int_{0}^{|z|} \mathrm{d} s / \varsigma(s)\right), z \in Q^{l}$, from (3.2) we have that

$$
\operatorname{Hess}^{M} \phi(x)(X, X)=\operatorname{Hess}^{Q} \zeta(z(x))\left(X_{Q}, X_{Q}\right)+\left\langle\operatorname{grad}^{Q} \zeta(z(x)), \alpha_{x}(X, X)\right\rangle
$$

for all vectors $X \in T_{X} M$, where $X_{Q}=z_{*} X$. Also observe that

$$
\operatorname{grad}^{Q} \zeta(z)=\frac{\zeta(z)}{\zeta(|z|)} \operatorname{grad}^{Q}|z|,
$$

and then

$$
\operatorname{grad}^{M} \phi(x)=\frac{\phi(x)}{\varsigma(|z(x)|)}\left(\operatorname{grad}^{Q}|z(x)|\right)^{T} .
$$


Thus, for every $X \in T_{x} M$ such that $X_{Q}=0$, it follows from (3.10) that

$$
\begin{aligned}
\operatorname{Hess}^{M} \phi(x)(X, X) & =\frac{\phi(x)}{\varsigma(|z(x)|)}\left\langle\operatorname{grad}^{Q}|z(x)|, \alpha_{x}(X, X)\right\rangle \\
& \leq \frac{\phi(x)}{\varsigma(|z(x)|)}\left\|\alpha_{x}(X, X)\right\| .
\end{aligned}
$$

Therefore, by (1.13) we obtain that

$$
\frac{1}{\phi(x)} \operatorname{Hess}^{M} \phi(x)(X, X) \leq \frac{\left\|\alpha_{x}(X, X)\right\|}{\varsigma(|z(x)|)} \leq\|X\|^{2}
$$

for every $X \in T_{x} M$ with $X_{Q}=0$. Given $W_{x_{k}} \subset T_{x_{k}} M$ with $\operatorname{dim} W_{x_{k}}>p+l$, we have that the subspace $V_{k}=f_{*}^{-1}\left(f_{*} W_{x_{k}} \cap T_{y_{k}} P\right)$ has $\operatorname{dim} V_{x_{k}} \geq \operatorname{dim} W_{x_{k}}-l>p$ and $f_{*}\left(V_{k}\right)$ is orthogonal to $T_{z_{k}} Q$. Then, $X_{Q}=0$ for every $X \in V_{x_{k}}$, and from (3.9) and (3.12) we get that

$$
\begin{aligned}
\operatorname{Hess}^{M} g\left(x_{k}\right)(X, X) & \leq \frac{g\left(x_{k}\right)-g\left(x_{0}\right)+1}{k \phi\left(x_{k}\right)} \operatorname{Hess}^{M} \phi\left(x_{k}\right)(X, X) \\
& \leq \frac{\psi_{b}(R)+1}{k}\|X\|^{2},
\end{aligned}
$$

for every $X \in V_{x_{k}}$. Moreover, using Theorem 11, we also have here that

$$
\operatorname{Hess}^{M} g(x)(X, X) \geq \psi_{b}^{\prime}(r(y(x)))\left(C_{b}(r(y(x)))\|X\|^{2}-\left\|\alpha_{x}(X, X)\right\|\right)
$$

for every $X \in V_{x_{k}}$, since $X_{P}=X$. Therefore, we obtain that

$$
\begin{aligned}
\frac{\psi_{b}(R)+1}{k}\|X\|^{2} & \geq \operatorname{Hess}^{M} g\left(x_{k}\right)(X, X) \\
& \geq \psi_{b}^{\prime}\left(r_{k}\right)\left(C_{b}\left(r_{k}\right)\|X\|^{2}-\left\|\alpha_{x_{k}}(X, X)\right\|\right)
\end{aligned}
$$

for every $x_{k}$ and every $X \in V_{x_{k}}$, where as usual $r_{k}=r\left(y_{k}\right)$. Hence

$$
\left\|\alpha_{x_{k}}(X, X)\right\| \geq\left(C_{b}\left(r_{k}\right)-\frac{\psi_{b}(R)+1}{k \psi_{b}^{\prime}\left(r_{k}\right)}\right)\|X\|^{2}
$$

with

$$
C_{b}\left(r_{k}\right)-\frac{\psi_{b}(R)+1}{k \psi_{b}^{\prime}\left(r_{k}\right)}>0
$$

for $k$ sufficiently large. Reasoning now as in the last part of the proof of Theorem 12, there exists a plane $\sigma_{k} \subset V_{x_{k}}$ such that, by the Gauss equation

$$
K_{f}\left(\sigma_{k}\right)=K_{\alpha}\left(\sigma_{k}\right) \geq\left(C_{b}\left(r_{k}\right)-\frac{\psi_{b}(R)+1}{k \psi_{b}^{\prime}\left(r_{k}\right)}\right)^{2},
$$

and (1.5) and (1.6) follow by letting $k \rightarrow+\infty$ as in the last part of the proof of Theorem 12. To finish the proof of Theorem 4 , we need to consider the case where the sequence $\left\{x_{k}\right\}_{k \in \mathbb{N}} \subset M^{m}$ remains in a compact set. In that case, passing to a subsequence if necessary, we may assume that $x_{k} \rightarrow x_{\infty} \in M^{m}$ and $g$ attains its absolute maximum at $x_{\infty}$. Thus $\operatorname{Hess}^{M} g\left(x_{\infty}\right)(X, X) \leq 0$ for all $X \in T_{x_{\infty}} M$. In particular, if follows from (3.13) that for every $X \in V_{x_{\infty}}$

$$
0 \geq \operatorname{Hess}^{M} g\left(x_{\infty}\right)(X, X) \geq \psi_{b}^{\prime}\left(r_{\infty}\right)\left(C_{b}\left(r_{\infty}\right)\|X\|^{2}-\left\|\alpha_{x_{\infty}}(X, X)\right\|\right) .
$$


Therefore

$$
\left\|\alpha_{x_{\infty}}(X, X)\right\| \geq C_{b}\left(r_{\infty}\right)\|X\|^{2} .
$$

By applying Lemma 1 to $\left.\alpha\right|_{V_{x_{\infty}} \times V_{x_{\infty}}}: V_{x_{\infty}} \times V_{x_{\infty}} \rightarrow N_{f} M\left(x_{\infty}\right)$ and reasoning again as in the last part of the proof of Theorem 12, we have that there exists a plane $\sigma_{\infty} \subset V_{x_{\infty}}$ such that, by the Gauss equation,

$$
K_{f}\left(\sigma_{\infty}\right)=K_{\alpha}\left(\sigma_{\infty}\right) \geq C_{b}^{2}\left(r_{\infty}\right),
$$

and (1.5) follows. Again (1.6) follows as in the last part of the proof of Theorem 12.

Proof of Theorem 5 We proceed as in the proof of Theorem 4 to obtain a sequence $\left\{x_{k}\right\}_{k \in \mathbb{N}}$ such that $g\left(x_{k}\right)$ converges to $g^{*}$ and satisfying

$$
\operatorname{grad}^{M} g\left(x_{k}\right)=\frac{g\left(x_{k}\right)-g\left(x_{0}\right)+1}{k \phi\left(x_{k}\right)} \operatorname{grad}^{M} \phi\left(x_{k}\right)
$$

and

$$
\operatorname{Hess}^{M} g\left(x_{k}\right) \leq \frac{g\left(x_{k}\right)-g\left(x_{0}\right)+1}{k \phi\left(x_{k}\right)} \operatorname{Hess}^{M} \phi\left(x_{k}\right) .
$$

Recall that [(see (3.11)]

$$
\operatorname{grad}^{M} \phi(x)=\frac{\phi(x)}{\varsigma(|z(x)|)}\left(\operatorname{grad}^{Q}|z(x)|\right)^{T} .
$$

Let us first consider the case where $x_{k} \rightarrow \infty$ as $k \rightarrow+\infty$. From (3.14) and (3.16) we know that

$$
\left\|\operatorname{grad}^{M} g\left(x_{k}\right)\right\| \leq \frac{g^{*}+1}{k} \frac{1}{\varsigma\left(\left|z_{k}\right|\right)} \leq \frac{\psi_{b}(R)+1}{k} \frac{1}{\varsigma\left(\left|z_{k}\right|\right)} .
$$

Since $f$ is proper and $\pi_{P}(f(M)) \subset B_{P}(R)$, then $\left|z_{k}\right| \rightarrow+\infty$ as $k \rightarrow+\infty$. Therefore, taking into account that $\lim \sup _{t \rightarrow+\infty} 1 / \varsigma(t)<+\infty$, we obtain from here that

$$
\lim _{k \rightarrow+\infty}\left\|\operatorname{grad}^{M} g\left(x_{k}\right)\right\|=0 \text {. }
$$

Observe that

$$
\operatorname{grad}^{N} h(f(x))=\psi_{b}^{\prime}(r(y)) \operatorname{grad}^{P} r(y)=\operatorname{grad}^{M} g(x)+\left(\operatorname{grad}^{N} h(f(x))\right)^{\perp},
$$

where $y=y(x)$. Therefore,

$$
\psi_{b}^{\prime}\left(r_{k}\right)^{2}=\left\|\operatorname{grad}^{M} g\left(x_{k}\right)\right\|^{2}+\left\|\left(\operatorname{grad}^{N} h\left(f\left(x_{k}\right)\right)\right)^{\perp}\right\|^{2},
$$

and making $k \rightarrow+\infty$ here we obtain that

$$
\lim _{k \rightarrow+\infty}\left\|\left(\operatorname{grad}^{N} h\left(f\left(x_{k}\right)\right)\right)^{\perp}\right\|=\psi_{b}^{\prime}\left(r^{*}\right)>0
$$

which implies that

$$
\left(\operatorname{grad}^{N} h\left(f\left(x_{k}\right)\right)\right)^{\perp} \neq 0
$$


for $k$ sufficiently large. As in the proof of Theorem 4, since $n-l>1$, given $W_{x_{k}} \subset T_{x_{k}} M$ with $\operatorname{dim} W_{x_{k}}>l+1$, we have that $V_{k}=f_{*}^{-1}\left(f_{*} W_{x_{k}} \cap T_{y_{k}} P\right)$ has $\operatorname{dim} V_{x_{k}} \geq \operatorname{dim} W_{x_{k}}-l>1$ and $f_{*}\left(V_{k}\right)$ is orthogonal to $T_{z_{k}} Q$. Then, using Theorem 11 , we also have that

$$
\operatorname{Hess}^{M} g\left(x_{k}\right)(X, X) \geq \psi_{b}^{\prime}\left(r_{k}\right)\left(C_{b}\left(r_{k}\right)\|X\|^{2}-\left\|\alpha_{x_{k}}(X, X)\right\|\right)
$$

for every $X \in V_{x_{k}}$, since $y_{*} X=X$. On the other hand, we also know from (3.15) that

$$
\begin{aligned}
\operatorname{Hess}^{M} g\left(x_{k}\right)(X, X) & \leq \frac{\psi_{b}(R)+1}{k} \frac{\operatorname{Hess}^{M} \phi\left(x_{k}\right)(X, X)}{\phi\left(x_{k}\right)} \\
& =\frac{\psi_{b}(R)+1}{k} \frac{1}{\varsigma\left(\left|z_{k}\right|\right)}\left\langle\operatorname{grad}^{Q}\left|z_{k}\right|, \alpha_{x_{k}}(X, X)\right\rangle
\end{aligned}
$$

for every $X \in T_{x_{k}} M$. Since we are in codimension one and $\left(\operatorname{grad}^{N} h\left(f\left(x_{k}\right)\right)\right)^{\perp} \neq 0$ (for $k$ large enough), then

$$
\alpha_{x_{k}}(X, X)=\lambda_{k}(X, X)\left(\operatorname{grad}^{N} h\left(f\left(x_{k}\right)\right)\right)^{\perp}
$$

for a real function $\lambda_{k}$. Now observe that

$$
\begin{aligned}
\left\langle\operatorname{grad}^{Q}\left|z_{k}\right|, \alpha_{x_{k}}(X, X)\right\rangle & =\lambda_{k}(X, X)\left\langle\operatorname{grad}^{Q}\left|z_{k}\right|,\left(\operatorname{grad}^{N} h\left(f\left(x_{k}\right)\right)\right)^{\perp}\right\rangle \\
& =\lambda_{k}(X, X)\left\langle\operatorname{grad}^{Q}\left|z_{k}\right|, \operatorname{grad}^{M} g\left(x_{k}\right)\right\rangle
\end{aligned}
$$

because of $\left\langle\operatorname{grad}^{Q}\left|z_{k}\right|, \operatorname{grad}^{P} r\left(y_{k}\right)\right\rangle=0$. Therefore,

$$
\begin{aligned}
\left\langle\operatorname{grad}^{Q}\left|z_{k}\right|, \alpha_{x_{k}}(X, X)\right\rangle & \leq\left|\lambda_{k}(X, X)\right|\left\|\operatorname{grad}^{M} g\left(x_{k}\right)\right\| \\
& \leq\left|\lambda_{k}(X, X)\right| \frac{\psi_{b}(R)+1}{k} \frac{1}{\varsigma\left(\left|z_{k}\right|\right)} .
\end{aligned}
$$

On the other hand, from our hypothesis (1.14) we know that

$$
\left\|\alpha_{x}(X, X)\right\| \leq \varsigma^{2}(|z(x)|)\|X\|^{2},
$$

and from (3.18) and (3.21) we have that

$$
\left\|\alpha_{x_{k}}(X, X)\right\|=\left|\lambda_{k}(X, X)\right| \sqrt{\psi_{b}^{\prime}\left(r_{k}\right)^{2}-\left\|\operatorname{grad}^{M} g\left(x_{k}\right)\right\|^{2}} \leq \varsigma^{2}\left(\left|z_{k}\right|\right)\|X\|^{2} .
$$

That is,

$$
\frac{\left|\lambda_{k}(X, X)\right|}{\varsigma\left(z_{k}\right)} \leq \frac{\varsigma\left(z_{k}\right)\|X\|^{2}}{\sqrt{\psi_{b}^{\prime}\left(r_{k}\right)^{2}-\left\|\operatorname{grad}^{M} g\left(x_{k}\right)\right\|^{2}}} .
$$

It follows from here that

$$
\left\langle\operatorname{grad}^{Q}\left|z_{k}\right|, \alpha_{x_{k}}(X, X)\right\rangle \leq \frac{\psi_{b}(R)+1}{k} \frac{\varsigma\left(z_{k}\right)\|X\|^{2}}{\sqrt{\psi_{b}^{\prime}\left(r_{k}\right)^{2}-\left\|\operatorname{grad}^{M} g\left(x_{k}\right)\right\|^{2}}}
$$

for every $X \in T_{x_{k}} M$, so that by (3.20) we get

$$
\operatorname{Hess}^{M} g\left(x_{k}\right)(X, X) \leq\left(\frac{\psi_{b}(R)+1}{k}\right)^{2} \frac{\|X\|^{2}}{\sqrt{\psi_{b}^{\prime}\left(r_{k}\right)^{2}-\left\|\operatorname{grad}^{M} g\left(x_{k}\right)\right\|^{2}}} .
$$


Therefore, from (3.19) and (3.22) we have that

$\psi_{b}^{\prime}\left(r_{k}\right)\left(C_{b}\left(r_{k}\right)\|X\|^{2}-\left\|\alpha_{x_{k}}(X, X)\right\|\right) \leq\left(\frac{\psi_{b}(R)+1}{k}\right)^{2} \frac{\|X\|^{2}}{\sqrt{\psi_{b}^{\prime}\left(r_{k}\right)^{2}-\left\|\operatorname{grad}^{M} g\left(x_{k}\right)\right\|^{2}}}$

for every $X \in V_{x_{k}}$. Hence

$$
\left\|\alpha_{x_{k}}(X, X)\right\| \geq\left(C_{b}\left(r_{k}\right)-\frac{\left(\psi_{b}(R)+1\right)^{2}}{k^{2} \psi_{b}^{\prime}\left(r_{k}\right) \sqrt{\psi_{b}^{\prime}\left(r_{k}\right)^{2}-\left\|\operatorname{grad}^{M} g\left(x_{k}\right)\right\|^{2}}}\right)\|X\|^{2},
$$

with

$$
\lim _{k \rightarrow+\infty}\left(C_{b}\left(r_{k}\right)-\frac{\left(\psi_{b}(R)+1\right)^{2}}{k^{2} \psi_{b}^{\prime}\left(r_{k}\right) \sqrt{\psi_{b}^{\prime}\left(r_{k}\right)^{2}-\left\|\operatorname{grad}^{M} g\left(x_{k}\right)\right\|^{2}}}\right)=C_{b}\left(r^{*}\right) \geq C_{b}(R)>0 .
$$

Reasoning now as in the last part of the proof of Theorem 12, there exists a plane $\sigma_{k} \subset V_{x_{k}}$ such that, by the Gauss equation,

$$
K_{f}\left(\sigma_{k}\right) \geq\left(C_{b}\left(r_{k}\right)-\frac{\left(\psi_{b}(R)+1\right)^{2}}{k^{2} \psi_{b}^{\prime}\left(r_{k}\right) \sqrt{\psi_{b}^{\prime}\left(r_{k}\right)^{2}-\left\|\operatorname{grad}^{M} g\left(x_{k}\right)\right\|^{2}}}\right)^{2},
$$

and (1.5) and (1.6) follow by letting $k \rightarrow+\infty$ as in the last part of the proof of Theorem 12. Finally, in the case where the sequence $\left\{x_{k}\right\}_{k \in \mathbb{N}} \subset M^{n}$ remains in a compact subset of $M^{n}$, and passing to a subsequence if necessary, we may assume that $x_{k} \rightarrow x_{\infty} \in M^{n}$ and $g$ attains its absolute maximum at $x_{\infty}$. Thus, $\operatorname{Hess}^{M} g\left(x_{\infty}\right)(X, X) \leq 0$ for all $X \in T_{x_{\infty}} M$. Therefore, it follows again from Theorem 11 that for every $X \in V_{x_{\infty}}$,

$$
0 \geq \operatorname{Hess}^{M} g\left(x_{\infty}\right)(X, X) \geq \psi_{b}^{\prime}\left(r_{\infty}\right)\left(C_{b}\left(r_{\infty}\right)\|X\|^{2}-\left\|\alpha_{x_{\infty}}(X, X)\right\|\right) .
$$

The proof now finishes as in Theorem 4 .

\section{Notes}

The idea of the proof that any compact surface in $\mathbb{R}^{3}$ must have a point of positive Gauss curvature was first taken up by Tompkins [20], who showed that there is no isometric immersion $f: M^{n} \rightarrow \mathbb{R}^{2 n-1}$ if $M^{n}$ is compact and flat. This result inspired the seminal paper of Chern-Kuiper [4], where Lemma 1 was proved for dimensions $n=2,3$ and conjectured to be true for any dimension. This conjecture was proved by Otsuki [13] for $\lambda=0$ who, consequently, obtained Theorem 6 for all dimensions.

The Chern and Kuiper result gave rise to a long series of works, among others, by O'Neill [12], Stiel [19], Moore [9], Jorge-Koutroufiotis [6], Pigola-Rigoli-Setti [14] and, finally, by Alías-Bessa-Montenegro [3] who obtained Theorem 1 on cylindrically bounded submanifolds.

The maximum principles used throughout this paper, as well as their related results, namely, Theorems 8, 9 and 10, are due to Pigola-Rigoli-Setti [14]. On the other hand, it was shown in [1] that conditions $(b)$ and $(c)$ in Theorem 8 can be replaced by the following equivalent although apparently stronger requirements:

(b) $\left\|\operatorname{grad}^{M} \gamma\right\| \leq c$ for a constant $c>0$ outside a compact subset of $M^{n}$, 
(c) $\operatorname{Hess}^{M} \gamma \leq d\langle$, $\rangle$ for a constant $d>0$ outside a compact subset of $M^{n}$.

A similar observation holds for the Omori-Yau maximum principle.

Regarding complete hypersurfaces of nonpositive Ricci curvature, Leung [8] pioneered their study by proving Theorem 3 in the case $b=0$ and conjecturing that the assumption on the sectional curvature could be dropped. This, however, turns out not to be true, as shown by Nadirashvili's [10] celebrated counterexample to both Hadamard's and CalabiYau's conjectures on negatively curved and minimal surfaces. After Leung's work, Smith [17] gave an answer for the case $b<0$ but with a non-sharp estimate (for having made the Hessian comparison to $\mathbb{R}^{n+1}$ instead of $\mathbb{H}_{b}^{n+1}$ ), and finally Veeravalli [21] obtained Theorem 3.

It is a natural question to ask whether Theorem 2 is still true in the limiting case, that is, when $R=\operatorname{inj}_{P}(o)=\frac{\pi}{2 \sqrt{b}}$, where $\frac{\pi}{2 \sqrt{b}}$ is replaced by $+\infty$ if $b \leq 0$. This motivates the following conjecture.

Conjecture 1 Let $f: M^{m} \rightarrow N^{n+l}=P^{n} \times Q^{l}$ be an isometric immersion with codimension $p=n+l-m<m-$ l of a complete Riemannian manifold. Assume that $R=\operatorname{inj}_{P}(o)=\frac{\pi}{2 \sqrt{b}}$, where $\frac{\pi}{2 \sqrt{b}}$ is replaced by $+\infty$ if $b \leq 0$. If $K_{P}^{\mathrm{rad}} \leq b$ in $B_{P}[R]$, then

$$
\sup _{M} \min \left\{\max _{\sigma \subset W} K_{f}(\sigma): \operatorname{dim} W>p+l\right\} \geq \max \{-b, 0\} .
$$

Moreover,

$$
\sup _{M} \min \left\{\max _{\sigma \subset W} K_{M}(\sigma): \operatorname{dim} W>p+l\right\} \geq \max \{-b, 0\}+\inf _{B_{P}[R]} K_{P} .
$$

It is not clear the extent to which the above conjecture is true, but an affirmative answer at least in the most classic cases, such as $P^{n}=\mathbb{R}^{n}$ and $l=0$, would have deep implications in the field of submanifolds with nonpositive extrinsic curvature. Indeed, Conjecture 1 in this case implies when $p=m-1$ that a complete Riemannian manifold $M^{m}$ with sectional curvature $K \leq-c<0$ cannot be immersed isometrically in $\mathbb{R}^{2 m-1}$, a kind of Efimov's theorem in $n$ dimensions. In particular, this would give us the $m$-dimensional version of the classical theorem of Hilbert that the hyperbolic plane cannot be realized isometrically in $\mathbb{R}^{3}$.

There is yet another attempt to extend Efimov's theorem to higher dimensions in a different direction proposed independently by Reilly [15] and Yau [22,23] (see also [24] and Gromov [5]):

"There are no complete hypersurfaces in $\mathbb{R}^{n+1}$ with Ricci curvature $\leq-c$ " and proved to be very true for $n=3$ and essentially true for $n>3$ by Smyth-Xavier [18]. Their main result seems to be inaccessible to techniques using the Omori-Yau maximum principle, and its proof relies on a purely geometric result on the principal curvatures of complete submanifolds of Euclidean space. Still in the case $P^{n}=\mathbb{R}^{n}$ and $l=0$, Conjecture 1 for $p=1$ would not only settle the above question at all, but also, in the same spirit of Corollary 3, weaken the assumption that Ric $\leq-c$ to $s \leq-c$.

\section{References}

1. Albanese, G., Alías, L.J., Rigoli, M.: A general form of the weak maximum principle and some applications. Rev. Mat. Iberoam. 29(4), 1437-1476 (2013)

2. Alías, L.J., Bessa, P., Dajczer, M.: The mean curvature of cylindrically bounded submanifolds. Math. Ann. 345(2), 367-376 (2009) 
3. Alías, L.J., Bessa, P., Fabio Montenegro, J.: An estimate for the sectional curvature of cylindrically bounded submanifolds. Trans. Am. Math. Soc. 364(7), 3513-3528 (2012)

4. Chern, S.-S., Kuiper, N.H.: Some theorems on the isometric imbedding of compact Riemann manifolds in Euclidean space. Ann. Math. 2(56), 422-430 (1952)

5. Gromov, M.: Partial differential relations. Ergebnisse der Mathematik und ihrer Grenzgebiete (3) [Results in mathematics and related areas (3)], vol. 9. Springer, Berlin (1986)

6. Jorge, L., Koutroufiotis, D.: An estimate for the curvature of bounded submanifolds. Am. J. Math. 103(4), 711-725 (1981)

7. Kobayashi, S., Nomizu, K.: Foundations of differential geometry. Vol. II. Wiley Classics Library. Wiley, New York (1996). (Reprint of the 1969 original, A Wiley-Interscience Publication)

8. Leung, P.F.: Complete hypersurface of nonpositive Ricci curvature. Bull. Aust. Math. Soc. 27(2), 215-219 (1983)

9. Moore, J.D.: An application of second variation to submanifold theory. Duke Math. J. 42, 191-193 (1975)

10. Nadirashvili, N.: Hadamard's and Calabi-Yau's conjectures on negatively curved and minimal surfaces. Invent. Math. 126(3), 457-465 (1996)

11. Omori, H.: Isometric immersions of Riemannian manifolds. J. Math. Soc. Jpn 19, $205-214$ (1967)

12. O'Neill, B.: Immersion of manifolds of nonpositive curvature. Proc. Am. Math. Soc. 11, 132-134 (1960)

13. Ôtsuki, T.: Isometric imbedding of Riemann manifolds in a Riemann manifold. J. Math. Soc. Jpn 6, 221-234 (1954)

14. Pigola, S., Rigoli, M., Setti, A.G.: Maximum principles on Riemannian manifolds and applications. Mem. Am. Math. Soc 174(822), x+99 (2005)

15. Reilly, R.C.: Applications of the Hessian operator in a Riemannian manifold. Indiana Univ. Math. J. 26(3), 459-472 (1977)

16. Schoen, R., Yau, S.-T.: Lectures on differential geometry. Conference Proceedings and Lecture Notes in Geometry and Topology, I. International Press, Cambridge, MA: Lecture notes prepared by Wei Yue Ding, Kung Ching Chang [Gong Qing Zhang], Jia Qing Zhong and Yi Chao Xu. Translated from the Chinese by Ding and S. Y, Cheng, Preface translated from the Chinese by Kaising Tso (1994)

17. Smith, G.H.: A note on complete hypersurfaces of nonpositive Ricci curvature. Bull. Aust. Math. Soc. 28(3), 339-342 (1983)

18. Smyth, B., Xavier, F.: Efimov's theorem in dimension greater than two. Invent. Math. 90(3), 443-450 (1987)

19. Stiel, E.F.: Immersions into manifolds of constant negative curvature. Proc. Am. Math. Soc. 18, 713-715 (1967)

20. Tompkins, C.: Isometric embedding of flat manifolds in Euclidean space. Duke Math. J. 5(1), 58-61 (1939)

21. Veeravalli, A.R.: A sharp lower bound for the Ricci curvature of bounded hypersurfaces in space forms. Bull. Aust. Math. Soc. 62(1), 165-170 (2000)

22. Yau, S.T.: Submanifolds with constant mean curvature. Am. J. Math 96, 346-366 (1974)

23. Yau, S.T.: Submanifolds with constant mean curvature. Am. J. Math 97, 76-100 (1975)

24. Yau, S.T. (ed.): Seminar on Differential Geometry, volume 102 of Annals of Mathematics Studies. In Papers presented at seminars held during the academic year 1979-1980. Princeton University Press, Princeton; University of Tokyo Press, Tokyo (1982) 